\section{Hemispheric mediation of same-different judgments}

\section{JUSTINE SERGENT \\ Montreal Neurological Institute, Montreal, Canada}

In a recent article, Bagnara, Boles, Simion, and Umilta (1983) reported two experiments aimed at examining the role of the cerebral hemispheres in the mediation of same-different judgments. The potential value of such an examination lies in the convergent information that may be derived about underlying processes, inasmuch as it has been suggested that (1) these matching operations represent basic mechanisms in human information processing (Krueger, 1978; Nickerson, 1978), (2) judgments of sameness and difference may involve independent and different perceptual operations (Bamber, 1969; Taylor, 1976), and (3) each hemisphere may be specialized for one type of judgment (Egeth \& Epstein, 1972). However, there is as yet no satisfactory explanation of the nature of same-different judgments in relation to the assumed processing competence of the cerebral hemispheres, and Bagnara et al.'s (1983) attempt to study further this relation seems to add to the puzzle. They used pairs of single letters, laterally presented simultaneously or successively in either visual field, and found significant interactions between visual field and type of match in both latency and accuracy data. They manipulated letter similarity and observed that this variable affected performance equally in both fields. In addition, the symmetry of the letters in "same" pairs improved performance in "same" matches. They suggested a visual comparison model that incorporates dual processing and response criterion elements to account for their findings. The purpose of this note is to comment on certain aspects of their approach and suggestions, and to point out some evidence that calls into question their conclusions. Two main aspects will be discussed in turn: the methodology and analyses, and the dual-process explanation of the findings.

\section{Methodology and Analyses}

Bagnara et al. (1983) presented a pair of uppercase letters (one above or to the side of the other in the simultaneous condition; one letter in the center followed $1,050 \mathrm{msec}$ later by one letter in either field in the successive condition), and had their subjects decide whether the two letters were the same or different in a manual reaction time (RT) task with the

The author's mailing address is: Montreal Neurological Institute, 3801 University Street, Montreal, PQ, Canada H3A 2B4. responding hands counterbalanced across subjects. One unusual aspect of the procedure was that "the subjects were allowed to view the display at selfselected distances," which Bagnara et al. (1983) justified by noting that "visual field asymmetry appears to be independent of eccentricity as long as displays are symmetric around central fixation" (p. 580). Although this justification is supported by the findings of some studies (e.g., Eriksen \& Schultz, 1977), it is contradicted by others (e.g., Lefton \& Haber, 1974, Experiment 1B; Sergent, 1983; Williams \& Lefton, 1981). In addition, varying the viewing distance also affects the visual angle subtended by the stimulus, and Pring (1981) found that the size of the stimulus could influence the pattern of visual-field asymmetries. As a consequence of this procedure, subjects were not exposed to the same viewing conditions, which introduces an unnecessary and uncontrolled source of variation within the group. Moreover, no information was provided about the range of selected viewing distances, which precludes comparison with other studies on this aspect. It may be that this range was small and without significant effect on performance, but invisible sources of variations in lateral tachistoscopic studies are too numerous to leave uncontrolled those variables that are readily identifiable.

Another uncontrolled source of variation in Bagnara et al.'s (1983) study was the responding hand, which was not included as a factor in the analyses. This is unfortunate, since there is evidence that this factor contributes to the pattern of visual-field asymmetries. Zaidel (1983), for example, has presented empirical findings and arguments pointing to the potential information that may be derived from analyzing the influence of the responding hand on visual-field performance. In addition, even when the responding hand is counterbalanced across subjects, one hand may be sufficient to determine the overall pattern of results, which implies that counterbalancing hands should not prevent examination of this factor. This is clearly illustrated in a study by Jonides (1979), who examined the effect of letter similarity on visual field performance in a manual RT task, as did Bagnara et al. (1983). Jonides (1979, Experiment 3) obtained a significant right visual field (RVF) superiority in "same" responses when target and nontarget letters were easily discriminable. However, this main effect was due uniquely to subjects' responding with their right hands, with subjects using their left hands showing a trend toward a left visual field (LVF) advantage. The influence of the responding hand is also illustrated in an earlier study by Bagnara, Boles, Simion, and Umiltà (1982), in which same-different judgments were examined as a function of the two visual fields. They then included re- 
sponding hand as well as key mapping as factors in their analyses and found these two variables to interact significantly with other variables, such as type of match and visual field. The failure to take the responding-hand factor into account in data analysis may thus prevent the identification of the exact nature of the significant interaction between type of match and visual field found by Bagnara et al. (1983). Although the responding hand does not always interact with visual field and type of match in visual laterality studies, such an absence of influence must be ascertained, especially when the "same" and "different" keys are assigned to different hands for each subject, as was the case in Bagnara et al.'s (1983) experiments.

\section{Dual-Process Comparison}

The empirical finding that "same" judgments are often made more quickly than "different" judgments has been a challenge to researchers interested in specifying the nature of the processes underlying these mental operations. Some authors have proposed that one single process mediates "same" and "different" judgments and have explained the RT disparity between these two judgments as the result of a priming effect when two "same" stimuli are presented (Proctor, 1981), as response competition affecting “different"' judgments (Eriksen, O'Hara, \& Eriksen, 1982), or as internal noise making it necessary to recheck "different" responses (Krueger, 1978). Most authors, however, have favored a dual-process matching, "same" comparisons being made by a fast holistic processor and "different" comparisons being made by a slow analytic processor (e.g., Bamber, 1969). None of these models can account for all existing findings, but the dual-process model often provides a satisfactory explanation of the results at the expense of parsimony.

Bagnara et al. (1983) opted for the dual-process model to explain the same-different RT disparity obtained in their experiments, and they suggested that only such a model could account for (1) the effect of symmetry in speeding "same" responses, (2) the demonstrated analytic nature of "different" judgments, and (3) the observed visual field $\times$ response interaction. In addition to logical difficulties inherent in a dual-process model, none of the three findings pointed out by Bagnara et al. seems compelling enough to warrant such a model.

On logical ground, a holistic-analytic dissociation to account for same-different judgments seems to run into difficulties. These two processes involve different strategies that must be implemented at the outset, but whether two stimuli are the same or different cannot be known before they are compared. This raises the question of how the processing organism will "know" whether to operate analytically or holistically; if it "knows," no comparison is nec- essary, since it implies that it has already obtained some information about the same or different nature of the pair. It may then be useful to examine the three findings that Bagnara et al. (1983) consider compelling in suggesting a dual-process model.

Role of symmetry. In Bagnara et al.'s (1983) experiments, symmetry was a characteristic manipulated in "same" pairs, and the results showed that both letter and display were determinant in speeding "same" responses. Since display symmetry is necessarily a property of "same" pairs, it becomes difficult to draw conclusions from such a finding with respect to a dual-process model. In addition, since Bagnara et al. (1983) did not examine whether "different" pairs made of symmetrical letters were processed faster thatn "different" pairs made of asymmetrical letters, it cannot be established that letter symmetry benefits only "same" comparisons.

More importantly, the finding that symmetry speeds "same" responses seems to be dependent on the particular letters composing the stimulus set. Egeth, Brownell, and Geoffrion (1976) found that one single particular pair (GG) was responsible for the slower matching of asymmetrical than of symmetrical pairs of uppercase letters (Experiments 3 and 4), and that symmetry per se was not the critical factor. The fact that Bagnara et al. (1983) included this particular pair in their set of asymmetrical letters may have biased the results toward the advantage of symmetry and may account for their finding. In addition, Bagnara et al. (1983) used Fox's (1975) suggestion, that symmetry might serve as a "diagnostic" allowing for fast "same" responses, to support their interpretation of the role of symmetry in letter matching. This is not justified, however, since Fox (1975) found no significant effects of symmetry in latency and errors when the stimuli were letters (Experiments 1 and 2), although the sign test was significant in the second experiment. It was only with nonalphanumeric visual patterns that Fox found a reliable effect of symmetry, and this finding cannot be generalized to letters, as demonstrated by Egeth et al. (1976). It is also true that when purely visual patterns are used, both "same" and "different" responses can be faster with symmetrical than with asymmetrical forms (Sebrechts \& Garner, 1981), although it must be noted that Hock (1973) found an effect of symmetry on "same" responses in only half his subjects. The role of symmetry in same-different judgments may thus not be as clear as that discussed by Bagnara et al. (1983), and it does not appear to be consistent with a dual-process model of letter matching.

Analytic nature of "different" comparisons. The view that "different" comparisons involve an analytic operation is based on the robust finding that $R T$ increases with a decrease in the number of differences between two stimuli (Bagnara et al., 1982; Bamber, 
1969; Smith \& Nielsen, 1970; Taylor, 1976). Whether such an increase in RT constitutes a valid criterion of an analytic operation is not guaranteed, however, and there are both theoretical and empirical reasons to question this interpretation.

Strictly speaking, an analytic operation implies the decomposition of stimuli into their components, and the matching of these components one by one. When the stimuli are single letters, the basic components are assumed to be the line segments (Bagnara et al., 1982; Taylor, 1976). On the other hand, when the stimuli are letter strings, the basic components are assumed to be single letters (e.g., Bamber, 1969). This raises the question of why an organism that is equipped to process such single letters as units should parse them into segments and therefore lose sight of their overall shape and identity. If the development of a percept unfolds from global configuration toward finer details as stimulus energy accumulates (e.g., Eriksen \& Schultz, 1979; Flavell \& Draguns, 1957), it is the configural shape of the letter that is first available, prior to the details, and the configuration may prove a sufficient basis for carrying out a comparison, at least for stimuli such as letters that can be resolved in a single glance. The configural dissimilarity between two letters is a function of the number of differences in line segments between them, but this does not necessarily imply that these line segments are processed separately and analytically. Instead, these particular line segments contribute to the configuration, and the configurally closer two letters are, as a function of their common segments, the slower it is to tell them apart (cf. Lockhead, 1972). An increase in RT with a decrease in the number of differences between two stimuli is therefore consistent with a configural mode of processing, which calls into question the so-called demonstrated analytic nature of "different" judgments. That is, two stimuli may not be compared in terms of their component dimensions treated independently of one another but rather in terms of their overall similiarity based on the configural shape that emerges from their component features or dimensions. For example, Munsell colors of constant hue varying in brightness and saturation are typically compared on the basis of their overall similarity and not as a function of their component dimensions (e.g., Garner, 1974; Hyman \& Well, 1967), yet it is faster to tell apart two colors if they differ on two dimensions than if they differ on only one (Sergent \& Takane, 1984). Unless an attempt is made to determine the rules by which the component dimensions are combined, there is no way of specifying the exact nature of the processes underlying "different" judgments.

Interaction between visual field and type of match. The third finding that led Bagnara et al. (1983) to suggest a dual-process model was the significant interaction between visual field and type of match.
They indicated that such an interaction implied a process dissociation by hemisphere, but it must be noted that the simple effects of this interaction were not significant. Bagnara et al. (1983) mentioned, nonetheless, that the obtained interaction was similar to others reported elsewhere (Bagnara et al., 1982; Cohen, 1973; Egeth \& Epstein, 1972), but a close examination of these studies should caution against such a suggestion.

Bagnara et al. (1982) conducted two experiments similar to the present ones and found that the interaction between visual field and type of match was "quite nonsignificant" in the analyses of latencies. In the analyses of errors, the interaction reached a reliable level of significance only in Experiment 2. These findings do not provide strong support for the view of a dissociation by hemisphere, which was, in fact, dismissed by the authors. Cohen (1973) presented letter strings in either visual field and had her subjects decide whether the strings were the same or different. Cohen (1973) did not directly test for the presence of a match $\times$ visual field interaction, since she analyzed "same" and "different" responses separately. In the two first experiments, she found that "same" judgments were made faster in the RVF than in the LVF, but no visual field effect emerged in "different" judgments. It must be noted that all the subjects responded "same" with their right hands and "different" with their left hands, which makes it difficult to determine the exact source of the effect. In fact, when responding hand was counterbalanced across subjects in the third experiment, there was no longer a field effect for "same" responses, and "different" responses were made faster in the LVF than in the RVF. Whether the latter effect obtained irrespective of the responding hand was not examined. Egeth and Epstein (1972) used a between-subject design and a go/no-go procedure, with one group responding only to "same" pairs and the other only to "different" pairs. Subjects in both groups used their "dominant" hands. The results showed no advantage of "same" over "different" responses, despite the exclusive use of symmetrical letters, which, according to Bagnara et al. (1983), can "dramatically speed 'same' responses." The interaction of type of match $\times$ visual field was significant for latencies ("same" faster in the RVF and "different" faster in the LVF), but subjects in the "different" group made more errors in the left than in the right visual field, suggesting a speed-accuracy tradeoff. Although the results of all these experiments might, at first examination, support Bagnara et al.'s (1983) suggestion, the findings are too ambiguous and the effects too variable to be used as a reliable confirmation of a dissociation by hemisphere.

There are, in fact, numerous studies in which such an interaction did not emerge and in which a LVF advantage prevailed for "same" judgments. This is 
probably best illustrated in research on faces, in which the matching of faces is often performed faster and more accurately in the LVF (see Ellis, 1983, for a review). In addition, damage to the right hemisphere tends to affect "same" judgments, whereas damage to the left hemisphere is more detrimental to "different" judgments (Buhr, 1983).

\section{Conclusion}

Bagnara et al. (1983) have suggested "a model of letter matching that can accommodate the results of Experiments 1 and 2," and this model seems to be only an ad hoc account of their particular findings. The specification of the processes underlying samedifferent judgments and of the nature of the processing specialization of the cerebral hemispheres is certainly a difficult task, and the considerable amount of data pertaining to these two fields of research is likely to preclude a unified explanation. This is not to say that such an attempt is bound to failure, but more control over the variables affecting performance and more stringent criteria to specify the nature of the underlying processes would be necessary to identify the particular mechanisms mediating samedifferent judgments in the two hemispheres.

\section{REFERENCES}

Bagnara, S., Boles, D. B., Simion, F., \& Umiltà, C. (1982). Can an analytic/holistic dichotomy explain hemispheric asymmetries? Cortex, 18, 67-78.

Bagnara, S., Boles, D. B., Simion, F., \& Umiltì, C. (1983). Symmetry and similarity effects in the comparison of visual patterns. Perception \& Psychophysics, 34, 578-584.

Bamber, D. (1969). Reaction times and error rate for samedifferent judgments of multidimensional stimuli. Perception \& Psychophysics, 6, 169-174.

Burr, R. D. (1983). Variations in "same"-"different" judgments in patients with unilateral cortical lesions. Neuropsychologia, 21, 607-615.

Conen, G. (1973). Hemispheric differences in serial versus parallel processing. Journal of Experimental Psychology, 97, 349-356.

Egeth, H. E., Browneli, H. H., \& Geoffrion, L. D. (1976). Testing the role of vertical symmetry in letter matching. Journal of Experimental Psychology: Human Perception and Performance, 2, 429-434.

Egeth, H. E., \& Epstein, J. (1972). Differential specialization of the cerebral hemispheres for the perception of sameness and difference. Perception \& Psychophysics, 12, 218-220.

Ellis, H. D. (1983). The role of the right hemisphere in face perception. In A. W. Young (Ed.), Functions of the right cerebral hemisphere (pp. 33-64). London: Academic Press.

Eriksen, C. W., O'Hara, W. P., \& Eriksen, B. A. (1982). Re- sponse competition effects in same-different judgments. Perception \& Psychophysics, 32, 262-270.

Eriksen, C. W., \& Schultz, D. W. (1977). Retinal locus and acuity in visual information processing. Bulletin of the Psychonomic Society, 9, 81-84.

ERIKsen, C. W., \& Schultz, D. W. (1979). Information processing in visual search: A continuous flow conception and experimental results. Perception \& Psychophysics, 25, 249-263.

Flavell, J., \& Draguns, J. (1957). A microgenetic approach to perception and thought. Psychological Bulletin, 54, 197-217.

Fox, J. (1975). The use of structural diagnostics in recognition. Journal of Experimental Psychology: Human Perception and Performance, 1, 57-67.

GARNER, W. R. (1974). The processing of information and structure. Potomac, MD: Erlbaum.

Hock, H. S. (1973). The effects of stimulus structure and familiarity on same-different judgments. Perception \& Psychophysics, 14, 413-420.

Hyman, R., \& Well, A. (1967). Judgments of similarity and spatial models. Perception \& Psychophysics, 2, 233-248.

JONIDES, J. (1979). Left and right visual field superiority for letter classification. Quarterly Journal of Experimental Psychology, 31, 423-439.

Krueger, L. (1978). A theory of perceptual matching. Psychological Review, 85, 278-304.

Lefton, L. A., \& Haber, R. N. (1974). Information extraction from different retinal locations. Journal of Experimental Psychology, 102, 975-980.

Lockhe AD, G. R. (1972). Processing dimensional stimuli: A note. Psychological Review, 79, 410-419.

Nicke RSON, R. S. (1978). On the time it takes to tell things apart. In J. Requin (Ed.), Attention and performance VII. Hillsdale, NJ: Erlbaum.

Pring, T. R. (1981). The effects of stimulus size and exposure duration on visual field asymmetries. Cortex, 17, 227-240.

Proctor, R. W. (1981). A unified theory for matching-task phenomena. Psychological Review, 88, 291-326.

Sebrechts, M. M., \& Garne R, W. R. (1981). Stimulus-specific processing consequences of pattern goodness. Memory \& Cognition, 9, 41-49.

Sergent, J. (1983). The effects of sensory limitations on hemispheric processing. Canadian Journal of Psychology, 37, 345-366.

Sergent, J., \& Takane, Y. (1984). Structure in two-choice reaction time. Manuscript in preparation.

Smith, E. E., \& Nielsen, G. D. (1970). Representation and retrieval processes in short term memory: Recognition and recall of faces. Journal of Experimental Psychology, 85, 397-405.

TAYLOR, D. A. (1976). Holistic and analytic processes in the comparison of letters. Perception \& Psychophysics, 20, 187-190.

Williams, L. J., \& LefTon, L. A. (1981). Processing of alphabetic information presented in the fovea or the periphery: Functional visual field or cognitive load. Perception, 10, 645-650.

ZaIDEL, E. (1983). Disconnection syndrome as a model for laterality effects in the normal brain. In J. B. Hellige (Ed.), Cerebral hemisphere asymmetry: Method, theory, and application (pp. 95-151). New York: Praeger Press.

(Manuscript received March 7, 1984;

revision accepted for publication April 24, 1984.) 